\title{
A Binary Classifier for Computing Posterior Error Probabilities in MetaMorpheus
}

Michael R. Shortreed ${ }^{1}$, Robert J. Millikin' ${ }^{1}$, Lei Liu ${ }^{1}$, Zach Rolfs ${ }^{1}$, Rachel M. Miller ${ }^{1}$, Leah V. Schaffer ${ }^{1}$, Brian L. Frey, ${ }^{1}$ Lloyd M. Smith ${ }^{1, *}$

${ }^{1}$ Department of Chemistry, University of Wisconsin-Madison, Madison, WI, USA ${ }^{*}$ Corresponding author. Tel: 1-608-263-2594. E-mail: smith@chem.wisc.edu

\section{Contents:}

Supplement 1: Extended figure caption for manuscript Figure 2. 


\section{Supplement 1.}

Figure 2. Explanation of the Axes. The vertical axis in each graph reports the relative fraction of True (target) identifications at the specified value $(T /(T+F))$, which scales between 0 and 1 . The horizontal axis for each graph covers the entire range of values for the labelled attribute (e.g. the values in the Total Matching Fragment Count graph are total matching fragment counts). The values plotted in the table are derived from the "AllPSMs_FormattedForPercolator.tab" file produced as output from every MetaMorpheus search. This table is produced in a manner that allows the same set of values used for the binary decision tree to be analyzed by percolator. Several values used in the binary decision tree have been length normalized including total matching fragment count, intensity, delta score, longest fragment ion series and complementary ion count. The PSM count is given the nearest value from the following list: $1,2,3,4,5,6,7,8,9,10,15,20,30,40,50,75,100,200,300,400$ and 500. The remaining attributes have non-normalized values. Normalization was used to improved stability in the computation of posterior error probability. 